QUARTERLY OF APPLIED MATHEMATICS

VOLUME LXVI, NUMBER 1

MARCH 2008, PAGES 123-137

S 0033-569X(07)01084-4

Article electronically published on December 6, 2007

\title{
$H^{\circ}$-TYPE RIEMANNIAN METRICS ON THE SPACE OF PLANAR CURVES
}

\author{
BY \\ JAYANT SHAH \\ Mathematics Department, Northeastern University, Boston, Massachusetts
}

\begin{abstract}
Michor and Mumford have shown that the distances between planar curves in the simplest metric (not involving derivatives) are identically zero. We derive geodesic equations and a formula for sectional curvature for conformally equivalent metrics. We show if the conformal factor depends only on the length of the curve, then the metric behaves like an $L^{1}$-metric, the sectional curvature is not bounded from above, and minimal geodesics may not exist. If the conformal factor is superlinear in curvature, then the sectional curvature is bounded from above.
\end{abstract}

1. Introduction. The purpose of this paper is to study the most basic properties of some of the simplest Riemannian metrics suggested by applications to computer vision. The problem is to understand and quantify similarities and differences between object shapes and their individual variations. At a fundamental level, the problem is to construct appropriate metrics on a space of closed surfaces in $\mathbb{R}^{3}$. A simpler version of the problem is the construction of Riemannian metrics on a space of closed planar curves. The choice of a metric depends on the type of similarity that is being considered. In their seminal paper [2], Michor and Mumford analyze two Riemannian metrics on a space of closed planar curves. Surprisingly, the Riemannian distance between any two curves in the simpler of the two metrics, an $H^{o}$-metric, turns out to be zero. To remedy this, they add a curvature term to the metric. Below, we analyze conformal variants of the $H^{\circ}$-metric of Michor and Mumford. After fixing the basic framework in $\S 2$, we investigate in $\S 3$ the existence of minimal geodesics in two specific cases where the conformal factor depends only on the length of the curve. We derive upper and lower bounds for distances between curves and show that these metrics behave like $L^{1}$-metrics. In the case of the simpler of the two metrics, the only minimal geodesics are those which deform the curve by moving all of its points with the same normal speed. In the case of the second metric, no geodesic is minimal if the length of the curve is less than a certain threshold; the

Received February 27, 2007.

2000 Mathematics Subject Classification. Primary 58E50; Secondary 53C22.

Key words and phrases. Moduli of planar curves, Differential geometry.

This work was supported by NIH Grant I-R01-NS34189-08.

E-mail address: shah@neu.edu

(C)2007 Brown University Reverts to public domain 28 years from publication 
question of minimality when the length of the deforming curve is equal to or greater than the threshold is still open. In $\S 4$, we derive an explicit geodesic equation and in $\S 5$, we provide a formula for the sectional curvature. We show that the sectional curvature is unbounded from above if the conformal factor depends only on the length of the curve. If the conformal factor is a superlinear function of the curvature, then the sectional curvature at a point is bounded with respect to all of the planes passing through a fixed tangent vector.

Conformal $H^{o}$-metrics using conformal factors depending on the length of the curve were proposed by Yezzi and Mennucci in [7], [8]. Higher-order metrics have also been proposed. Younes has proposed an $H^{1}$-metric in [9]. Mio et al [4], [5], [6] have constructed geodesics in $H^{1}$ - and $H^{2}$-metrics. More recently, Michor and Mumford have described a general Hamiltonian framework for studying Sobolev metrics on the space of planar curves [3].

2. The framework. The basic space considered by Michor and Mumford is the orbit space

$$
B_{e}\left(S^{1}, \mathbb{R}^{2}\right)=\operatorname{Emb}\left(S^{1}, \mathbb{R}^{2}\right) / \operatorname{Diff}\left(S^{1}\right)
$$

of the space of all $C^{\infty}$ embeddings of $S^{1}$ in the plane, under the action by composition from the right by diffeomorphisms of the unit circle. It is contained in the bigger space of immersions modulo diffeomorphisms:

$$
B_{i}\left(S^{1}, \mathbb{R}^{2}\right)=\operatorname{Imm}\left(S^{1}, \mathbb{R}^{2}\right) / \operatorname{Diff}\left(S^{1}\right) .
$$

Let $\pi: \operatorname{Imm}\left(S^{1}, \mathbb{R}^{2}\right) \rightarrow B_{i}\left(S^{1}, \mathbb{R}^{2}\right)$ be the canonical projection. The simpler of the two metrics considered in [2] is an $H^{o}$-metric defined on $\operatorname{Imm}\left(S^{1}, \mathbb{R}^{2}\right)$ :

$$
G_{c}^{o}(m, h)=\int_{S^{1}}(m \cdot h)\left|c_{\theta}\right| d \theta
$$

where $c: S^{1} \rightarrow \mathbb{R}^{2}$ is an immersion, defining a point in $\operatorname{Imm}\left(S^{1}, \mathbb{R}^{2}\right), m, h \in C^{\infty}\left(S^{1}, \mathbb{R}^{2}\right)$ are the vector fields along the image curve, defining two tangent vectors on $\operatorname{Imm}\left(S^{1}, \mathbb{R}^{2}\right)$ at $c$, and $c_{\theta}=d c / d \theta .(m \cdot h)$ is the usual dot product in $\mathbb{R}^{2}$. Sometimes, for the sake of clarity, we will use the notation $a \cdot b$ even when $a, b$ are scalars. Let $n_{c}$ denote the unit normal field along $c$. If we identify $\mathbb{R}^{2}$ with the complex plane $\mathbb{C}$, then $n_{c}=i c_{\theta} /\left|c_{\theta}\right|$. The tangent vectors on $B_{i}\left(S^{1}, \mathbb{R}^{2}\right)$ at $\pi(c)$ are of the form $a n_{c}$ where $a \varepsilon C^{\infty}\left(S^{1}, \mathbb{R}\right)$. For any $C_{o}, C_{1} \in B_{i}$, consider all liftings $c_{o}, c_{1}$ to $\operatorname{Imm}\left(S^{1}, \mathbb{R}^{2}\right)$ and all smooth paths $t \mapsto(\theta \mapsto c(t, \theta)), 0 \leq t \leq 1$, in $\operatorname{Imm}\left(S^{1}, \mathbb{R}^{2}\right)$ with $c(0, \cdot)=c_{o}$ and $c(1, \cdot)=c_{1}$. Let $c_{t}$ denote $\partial c / \partial t$ and $c_{t}^{\perp}=\left(c_{t} \cdot n_{c}\right) n_{c}$. The arclength of such a path $c$ is given by

$$
\int_{0}^{1} \sqrt{G_{c}^{o}\left(c_{t}, c_{t}\right)} d t
$$

Michor and Mumford show that for any two curves in $B_{i}\left(S^{1}, \mathbb{R}^{2}\right)$,

$$
\operatorname{dist}_{G^{o}}\left(C_{1}, C_{2}\right)=\operatorname{def}_{c} \inf _{c} \int_{0}^{1} \sqrt{G_{c}^{o}\left(c_{t}^{\perp}, c_{t}^{\perp}\right)} d t=0
$$


and they strengthen $G^{o}$ by defining

$$
G_{c}^{A}(m, h)=\int_{S^{1}}\left(1+A \kappa_{c}^{2}\right)(m \cdot h)\left|c_{\theta}\right| d \theta
$$

where $\kappa_{c}$ is the curvature, defined by the equation $\left(\frac{c_{\theta}}{\left|c_{\theta}\right|}\right)_{\theta}=i \kappa_{c} c_{\theta}=\kappa_{c}\left|c_{\theta}\right| n_{c}$.

An alternative is to consider conformal transformations of $G^{o}$ :

$$
G_{c}^{\rho}(m, h)=\rho(c) \int_{S^{1}}(m \cdot h)\left|c_{\theta}\right| d \theta,
$$

where $\rho(c)$ is a $\operatorname{Diff}\left(S^{1}\right)$-invariant function on $\operatorname{Imm}\left(S^{1}, \mathbb{R}^{2}\right)$. In this paper, we consider conformal factors $\rho$ that are functions of the curve length $\ell$ and the curvature $\kappa$.

Notation. Throughout this paper, we will use the superscript $\rho$ (respectively $o$ ) to label quantities that are calculated using the metric $G^{\rho}$ (respectively $G^{o}$ ), except that $G_{c}^{\rho}(h, h)$ will be denoted as $\|h\|_{\rho}^{2}$ and $G^{o}(h, h)$ simply as $\|h\|^{2}$.

3. Instability of $G^{\rho(\ell)}$ geodesics. In this section, we assume that $\rho$ is a function of the curve length $\ell$ alone: $\rho(c)=\ell(c)$ or $e^{A \ell(c)}$ where $A$ is a positive constant. If $c$ is a path connecting curves $C, C^{\prime}$, let $\alpha(c)$ denote the area swept out by $c$ in $\mathbb{R}^{2}$. For a path $c(t, \cdot)$, let $\ell_{\max }(c)=\max _{t} \ell(c(t, \cdot))$. The following theorem characterizes the $L^{1}$-type behavior of the metrics $G^{\rho(\ell)}$.

Theorem 3.1. If $\rho(c)=\ell(c)$, then

$$
\operatorname{dist}_{G^{\rho}}\left(C, C^{\prime}\right)=\inf _{c} \alpha(c) .
$$

If $\rho(c)=e^{A \ell(c)}$, then

$$
\inf _{c} \sqrt{A e} \alpha(c) \leq \operatorname{dist}_{G^{\rho}}\left(C, C^{\prime}\right) \leq \inf _{c} \sqrt{A e} e^{A \ell_{\max }(c) / 2} \alpha(c) .
$$

We first prove a series of lemmas.

LEMMA 3.2 .

$$
\operatorname{dist}_{G^{\rho}}\left(C, C^{\prime}\right) \geq\left\{\begin{array}{l}
\inf _{c} \alpha(c) \text { if } \rho(c)=\ell(c), \\
\inf _{c} \sqrt{A e} \alpha(c) \text { if } \rho(c)=e^{A \ell(c)} .
\end{array}\right.
$$

Proof. For any path $c$,

$$
\begin{aligned}
L_{G^{\rho}}(c) & =\int_{0}^{1}\left[\rho(c) \int_{S^{1}}\left(c_{t}^{\perp} \cdot c_{t}^{\perp}\right)\left|c_{\theta}\right| d \theta\right]^{\frac{1}{2}} d t \\
& \geq \int_{0}^{1}\left[\left(\frac{\rho(c)}{\left|\sup \left(c_{t}^{\perp}\right)\right|}\right)^{\frac{1}{2}} \int_{S^{1}}\left|c_{t}^{\perp}\right|\left|c_{\theta}\right| d \theta\right] d t \\
& \geq\left[\min _{t} \frac{\rho(c)}{\ell(c)}\right]^{\frac{1}{2}} \int_{S^{1} \times[0,1]}|\operatorname{det} d c(t, \theta)| d \theta d t \\
& \geq\left\{\begin{array}{l}
\alpha(c) \text { if } \rho(c)=\ell(c), \\
\sqrt{A e} \alpha(c) \text { if } \rho(c)=e^{A \ell(c)} .
\end{array}\right.
\end{aligned}
$$


David Mumford observed from the formula for the sectional curvature that the geodesics along which $\left|\sup \left(c_{t}^{\perp}\right)\right|<\ell$ if $\rho=\ell$ and $<1 / A$ if $\rho=e^{A \ell}$ may not be minimal. Such a possibility can be heuristically seen from the inequality

$$
L_{G^{\rho}}(c) \geq \int_{0}^{1}\left[\left(\frac{\rho(c)}{\left|\sup \left(c_{t}^{\perp}\right)\right|}\right)^{\frac{1}{2}} \int_{S^{1}}\left|c_{t}^{\perp}\right|\left|c_{\theta}\right| d \theta\right] d t,
$$

which suggests that while traversing a given area, one should try to minimize $\rho(c) /\left|\sup \left(c_{t}^{\perp}\right)\right|$. The key point is that we can increase $\left|\sup \left(c_{t}^{\perp}\right)\right|$ indefinitely by replacing the part of the curve supporting $c_{t}^{\perp}$ by a saw-tooth-shaped curve of high frequency and small amplitude. When $\rho=\ell$ and $\left|\sup \left(c_{t}^{\perp}\right)\right|<\ell$, we can increase $\left|\sup \left(c_{t}^{\perp}\right)\right|$ so that $\ell /\left|\sup \left(c_{t}^{\perp}\right)\right|$ tends to 1 . When $\rho=e^{A \ell}$ and $\left|\sup \left(c_{t}^{\perp}\right)\right|<1 / A$, we can force $e^{A\left|\sup \left(c_{t}^{\perp}\right)\right|} /\left|\sup \left(c_{t}^{\perp}\right)\right|$ to equal its unique minimum $A e$ by making $\left|\sup \left(c_{t}^{\perp}\right)\right|$ equal to $1 / A$. (In the case of the metric $G^{o}, \rho=1$ so that $\rho /\left|\sup \left(c_{t}^{\perp}\right)\right|$ tends to 0 .) In order to obtain an upper bound for a general path, we break it up into a series of tiny bumps. When $\rho=\ell$, this method gives an upper bound for $\operatorname{dist}_{G^{\rho}}\left(C, C^{\prime}\right)$ that coincides with the lower bound. When $\rho=e^{A \ell}$, the larger the value of $\left|\sup \left(c_{t}^{\perp}\right)\right|$, the greater the divergence between the upper bound obtained by this method and the lower bound since it is more efficient to create a large bump all at once rather than a series of tiny bumps.

\section{Rectangular Bumps.}

Let $c_{o}: S^{1} \rightarrow \mathbb{R}^{2}$ be a smooth and free immersion. Let $C_{o}$ be the corresponding curve in $\mathbb{R}^{2}$. Let $c_{o}$ be parametrized by the arclength so that $\theta$ parametrizes the scaled circle $S_{\ell_{o}}^{1}$, where $\ell_{o}$ is the length of $C_{o}$. For any function $u(\theta)$, let $u^{\prime}$ denote $d u / d \theta$. Let $n_{o}$ denote the normal vector $i c_{o}^{\prime}$. Let $\kappa_{o}$ denote the curvature of $c_{o}$.

Fix small positive numbers $\delta$ and $\epsilon$ such that $\delta<\ell_{o}$ and $\epsilon\left\|\kappa_{o}\right\|_{\infty,[0, \delta]}<<1$. Construct a "rectangular" bump over $C_{o}$ as follows:

$$
c_{1}(\theta)= \begin{cases}c_{o}(\theta)+\epsilon n_{o} & \text { if } 0<\theta<\delta \\ \left\{c_{o}(\theta)+s n_{o} \mid 0 \leq s \leq \epsilon\right\} & \text { if } \theta=0, \delta \\ c_{o}(\theta) & \text { otherwise }\end{cases}
$$

Let $C_{1}$ be the corresponding curve in $\mathbb{R}^{2}$.

Lemma 3.3. For a rectangular bump $C_{1}$ over a curve $C_{o}$, we have the following estimates:

(i) If $\rho=\ell$,

$$
\operatorname{dist}_{G^{\rho}}\left(C_{o}, C_{1}\right) \leq\left[\frac{1+\epsilon\left\|\kappa_{o}\right\|_{\infty,[o, \delta]}}{1-\epsilon\left\|\kappa_{o}\right\|_{\infty,[o, \delta]}}\right]^{2} \text { (area of the bump). }
$$

(ii) If $\rho=e^{A \ell}$ and $\delta<1 / A$,

$$
\begin{aligned}
& \operatorname{dist}_{G^{\rho}}\left(C_{o}, C_{1}\right) \\
& \leq\left[\frac{1+\epsilon|| \kappa_{o} \|_{\infty,[o, \delta]}}{1-\epsilon|| \kappa_{o} \|_{\infty,[o, \delta]}}\right]^{3 / 2} e^{A\left(\ell_{o}+2 \epsilon-\delta\right) / 2} \sqrt{A e^{\frac{1+\epsilon|| \kappa_{o}\|\|_{\infty,[o, \partial]}}{1-\epsilon \mid \kappa_{o}\|\|_{\infty,[o, \partial]}}} \text { (bump area). }}
\end{aligned}
$$

Proof. We prove the lemma using a modification of the "teeth" construction of Michor and Mumford [2]. If $\rho=\ell$, choose $A<1 / \delta$. Approximate $C_{1}$ by a "trapezoidal" bump $\tilde{C}_{1}$ as follows. Replace $C_{0}$ in the interval $[0, \delta]$ by a saw-tooth curve of height $\eta$ and period $\frac{1}{m}$ such that its length equals $\frac{1}{A}$. This is done by growing teeth on $C_{0}$ in time 
$\eta$. Move the saw-tooth curve at unit speed along the normals $n_{0}$ keeping its end-points fixed, until it touches the upper edge of the bump. Finally, retract the teeth in time $\eta$. Formally, define a path $c(t, \theta)=c_{o}(\theta)+f(t, \theta) n_{o}$ where $f(t, \theta)$ is defined as follows:

$$
f(t, \theta)=0,0 \leq t \leq \epsilon \text { and } \delta \leq \theta \leq \ell_{o} .
$$

For $0 \leq t \leq \eta, 0 \leq k \leq m-1$,

$$
f(t, \theta)= \begin{cases}t\left(\frac{2 m \theta}{\delta}-2 k\right) & \frac{2 k}{2 m} \leq \frac{\theta}{\delta} \leq \frac{2 k+1}{2 m} \\ t\left(2 k+2-\frac{2 m \theta}{\delta}\right) & \frac{2 k+1}{2 m} \leq \frac{\theta}{\delta} \leq \frac{2 k+2}{2 m}\end{cases}
$$

For $\eta \leq t \leq \epsilon-\eta$,

$$
f(t, \theta)= \begin{cases}\frac{\epsilon(t-\eta)+\eta(\epsilon-\eta-t)}{\epsilon-2 \eta} \cdot \frac{2 m \theta}{\delta} & 0 \leq \frac{\theta}{\delta} \leq \frac{1}{2 m} \\ \frac{\epsilon-\eta}{\epsilon-2 \eta}(t-\eta)+f(\eta, \theta) & \frac{1}{2 m} \leq \frac{\theta}{\delta} \leq 1-\frac{1}{2 m} \\ \frac{\epsilon(t-\eta)+\eta(\epsilon-\eta-t)}{\epsilon-2 \eta} \cdot 2 m\left(1-\frac{\theta}{\delta}\right) & 1-\frac{1}{2 m} \leq \frac{\theta}{\delta} \leq 1 .\end{cases}
$$

For $\epsilon-\eta \leq t \leq \epsilon$

$$
\begin{aligned}
f(t, \theta) & = \begin{cases}\frac{2 m \epsilon \theta}{\delta} & 0 \leq \frac{\theta}{\delta} \leq \frac{1}{2 m} \\
\frac{\epsilon[t-(\epsilon-\eta)]+(\epsilon-t) f(\epsilon-\eta, \theta)}{\eta} & \frac{1}{2 m} \leq \frac{\theta}{\delta} \leq 1-\frac{1}{2 m} \\
2 m \epsilon\left(1-\frac{\theta}{\delta}\right) & 1-\frac{1}{2 m} \leq \frac{\theta}{\delta} \leq 1 .\end{cases} \\
c^{\prime} & =c_{o}^{\prime}+f^{\prime} n_{o}-f \kappa_{o} c_{o}^{\prime}=\left(1-f \kappa_{o}\right) c_{o}^{\prime}+f^{\prime} n_{o} \\
\left|c^{\prime}\right| & =\sqrt{\left(1-f \kappa_{o}\right)^{2}+f^{\prime 2}} \\
n & =\frac{-f^{\prime} c_{o}^{\prime}+\left(1-f \kappa_{o}\right) n_{o}}{\left|c^{\prime}\right|} \\
c_{t} & =f_{t} n_{o} \\
c_{t}^{\perp} \cdot c_{t}^{\perp} & =\left(c_{t} \cdot n\right)^{2}=\frac{\left(1-f \kappa_{o}\right)^{2} f_{t}^{2}}{\left|c^{\prime}\right|^{2}} .
\end{aligned}
$$

Let

$$
\begin{aligned}
& \beta=\sqrt{\frac{\left(1+\epsilon|| \kappa_{o} \|_{\infty,[o, \delta]}\right)^{2}+f^{\prime 2}}{\left(1-\epsilon|| \kappa_{o} \|_{\infty,[o, \delta]}\right)^{2}+f^{\prime 2}}} \\
& 1 \leq \beta \leq \frac{1+\epsilon|| \kappa_{o} \|_{\infty,[o, \delta]}}{1-\epsilon|| \kappa_{o} \|_{\infty,[o, \delta]}}
\end{aligned}
$$

Choose $m$ and $\eta$ such that $\int_{o}^{\delta}\left|c^{\prime}(\eta, \theta)\right| d \theta=\frac{1}{A}$. Note that as $m \rightarrow \infty, \eta \rightarrow 0$.

Estimates when $0 \leq t \leq \eta$ : Since $\left|f^{\prime}\right|$ is independent of $\theta$ and $|f| \leq \epsilon$,

$$
\begin{gathered}
\sqrt{\left(1-\epsilon|| \kappa_{o} \|_{\infty,[o, \delta]}\right)^{2}+f^{\prime 2}} \leq\left|c^{\prime}(\eta, \theta)\right| \leq \sqrt{\left(1+\epsilon\left\|\kappa_{o}\right\|_{\infty,[o, \delta]}\right)^{2}+f^{\prime 2}} \\
\delta \sqrt{\left(1-\epsilon\left\|\kappa_{o}\right\|_{\infty,[o, \delta]}\right)^{2}+f^{\prime 2}} \leq \int_{o}^{\delta}\left|c^{\prime}(\eta, \theta)\right| d \theta=\frac{1}{A} \leq \delta \sqrt{\left(1+\epsilon\left\|\kappa_{o}\right\|_{\infty,[o, \delta]}\right)^{2}+f^{\prime 2}} .
\end{gathered}
$$


We also have

$$
\begin{aligned}
\sqrt{\left(1-\epsilon\left\|\kappa_{o}\right\|_{\infty,[o, \delta]}\right)^{2}+f^{\prime 2}} & \leq\left|c^{\prime}(t, \theta)\right| \leq \sqrt{\left(1+\epsilon|| \kappa_{o} \|_{\infty,[o, \delta]}\right)^{2}+f^{\prime 2}} \\
\frac{1}{\beta} \sqrt{\left(1+\epsilon\left\|\kappa_{o}\right\|_{\infty,[o, \delta]}\right)^{2}+f^{\prime 2}} & \leq\left|c^{\prime}(t, \theta)\right| \leq \beta \sqrt{\left(1-\epsilon|| \kappa_{o} \|_{\infty,[o, \delta]}\right)^{2}+f^{\prime 2}} \\
\frac{1}{\beta} \cdot \frac{1}{A \delta} & \leq\left|c^{\prime}(t, \theta)\right| \leq \frac{\beta}{A \delta} \\
\ell(c)=\int_{o}^{\ell_{o}}\left|c^{\prime}\right| d \theta & \leq\left(\ell_{o}-\delta\right)+\frac{\beta}{A} \\
e^{A \ell(c)} & \leq e^{A\left(\ell_{o}-\delta\right)} e^{\beta} .
\end{aligned}
$$

Since $\left|f_{t}\right| \leq 1$

$$
\begin{gathered}
\int_{o}^{\ell_{o}}\left|c_{t}^{\perp}\right|^{2}\left|c^{\prime}\right| d \theta=\int_{o}^{\delta} \frac{\left(1-f \kappa_{o}\right)^{2} f_{t}^{2}}{\left|c^{\prime}\right|} d \theta \leq\left(1+\epsilon\left\|\kappa_{o}\right\|_{\infty,[o, \delta]}\right)^{2} \beta A \delta^{2} \\
\lim _{m \rightarrow \infty} \int_{o}^{\eta}\left[\rho(\ell(c)) \int_{o}^{\ell_{o}}\left|c_{t}^{\perp}\right|^{2}\left|c^{\prime}\right| d \theta\right]^{1 / 2} d t=0 .
\end{gathered}
$$

Estimates when $\eta \leq t \leq \epsilon-\eta$ : The estimate for $\left|c^{\prime}(t, \theta)\right|$ is the same as in the interval $\left[\frac{\delta}{2 m}, \delta\left(1-\frac{1}{2 m}\right)\right]$ since the curve has the same shape. In the intervals $\left[0, \frac{\delta}{2 m}\right]$ and $\left[\delta\left(1-\frac{1}{2 m}\right), \delta\right], \frac{2 m \eta}{\delta} \leq\left|f^{\prime}(t, \theta)\right| \leq \frac{2 m \epsilon}{\delta}$. Therefore, $\left|c^{\prime}(\eta, \theta)\right| \leq\left|c^{\prime}(t, \theta)\right| \leq 1+\epsilon|| \kappa_{o} \|_{\infty,[o, \partial]}+$ $\frac{2 m \epsilon}{\delta}$.

$$
\begin{aligned}
& \ell(c)=\int_{o}^{\ell_{o}}\left|c^{\prime}\right| d \theta \leq\left(\ell_{o}-\delta\right)+\left(1+\epsilon\left\|\kappa_{o}\right\|_{\infty,[o, \delta]}+\frac{2 m \epsilon}{\delta}\right) \frac{\delta}{m}+\frac{\beta}{A} \\
& \lim _{m \rightarrow \infty} \ell(c) \leq\left(\ell_{o}-\delta\right)+2 \epsilon+\frac{1+\epsilon\left\|\kappa_{o}\right\|_{\infty,[o, \delta]}}{1-\epsilon\left\|\kappa_{o}\right\|_{\infty,[o, \delta]}} \frac{1}{A} \\
& \lim _{m \rightarrow \infty} e^{A \ell(c)} \leq e^{\left(A \ell_{o}+2 \epsilon-\delta\right)} e^{\frac{1+\epsilon\|\|_{o} \|_{\infty,[o, \delta]}}{1-\epsilon \mid \kappa_{o} \|_{\infty,[o, \delta]}}} .
\end{aligned}
$$

We also have $\left|f_{t}\right| \leq \frac{\epsilon-\eta}{\epsilon-2 \eta}$. Therefore,

$$
\begin{aligned}
& \int_{o}^{\ell_{o}}\left|c_{t}^{\perp}\right|^{2}\left|c^{\prime}\right| d \theta \leq\left(1+\epsilon\left\|\kappa_{o}\right\|_{\infty,[o, \delta]}\right)^{2}\left(\frac{\epsilon-\eta}{\epsilon-2 \eta}\right)^{2} \beta A \delta^{2} \\
& \lim _{m \rightarrow \infty} \int_{\eta}^{\epsilon-\eta}\left[\rho(\ell(c)) \int_{o}^{\ell_{o}}\left|c_{t}^{\perp}\right|^{2}\left|c^{\prime}\right| d \theta\right]^{1 / 2} d t \\
& \leq\left\{\begin{array}{l}
\left(1+\epsilon\left\|\kappa_{o}\right\|_{\infty,[o, \delta]}\right) \sqrt{\frac{1+\epsilon\left\|\kappa_{o}\right\|_{\infty,[o, \delta]}}{1-\epsilon|| \kappa_{o} \|_{\infty,[o, \delta]}}}\left[A\left(\ell_{o}+2 \epsilon-\delta\right)+\frac{1+\epsilon\left\|\kappa_{o}\right\|_{\infty,[o, \delta]}}{1-\epsilon\left\|\kappa_{o}\right\|_{\infty,[o, \delta]}}\right]^{1 / 2} \delta \epsilon \\
\text { if } \rho(\ell)=\ell, \\
\left(1+\epsilon\left\|\kappa_{o}\right\|_{\infty,[o, \delta]}\right) \sqrt{\frac{1+\epsilon\left\|\kappa_{o}\right\|_{\infty,[o, \delta]}}{1-\epsilon\left\|\kappa_{o}\right\|_{\infty,[o, \delta]}}} e^{A\left(\ell_{o}+2 \epsilon\right) / 2} \sqrt{A e^{\frac{1+\epsilon \mid \kappa_{o} \|_{\infty,[o, \delta]}}{1-\epsilon \mid \kappa_{o} \|_{\infty,[o, \delta]}}-A \delta}} \delta \epsilon \\
\text { if } \rho(\ell)=e^{A \ell} .
\end{array}\right.
\end{aligned}
$$


Estimates when $\epsilon-\eta \leq t \leq \epsilon$ : The path $c(t, \theta)$ in the interval $[\epsilon-\eta, \epsilon]$ is essentially the same as that in $[0, \eta]$ and

$$
\lim _{m \rightarrow \infty} \int_{\epsilon-\eta}^{\epsilon}\left[\rho(\ell(c)) \int_{o}^{\ell_{o}}\left|c_{t}^{\perp}\right|^{2}\left|c^{\prime}\right| d \theta\right]^{1 / 2} d t=0 .
$$

Since

$$
\text { (area of the bump) }=\int_{o}^{\epsilon} \int_{o}^{\delta}\left(1-t \kappa_{o}\right) d \theta d t \geq\left(1-\epsilon\left\|\kappa_{o}\right\|_{\infty,[o, \delta]}\right) \delta \epsilon,
$$

the lemma is proved in the case when $\rho=e^{A \ell}$. It is proved in the case when $\rho=\ell$ by letting $A \rightarrow 0$.

In Lemma 3.3, we may replace the single bump by a finite number of disjoint bumps of height $\epsilon$ and total length $\delta$. The proof remains unchanged except that we must replace $2 \epsilon$ in the formula by $2 k \epsilon$ if $k$ is the number of bumps. The function $f$ in each individual bump may be positive or negative.

We prove the theorem by approximating the path by a series of small rectangular bumps. The error of approximation may be made arbitrarily small by the following lemma. Define a Fréchet metric on $\operatorname{Imm}\left(S^{1}, \mathbb{R}^{2}\right)$ and $B_{i}\left(S^{1}, \mathbb{R}^{2}\right)$ as follows. If $c_{o}, c_{1}$ are points in $\operatorname{Imm}\left(S^{1}, \mathbb{R}^{2}\right)$, let

$$
d_{\infty}\left(c_{o}, c_{1}\right)=\sup _{\theta}\left|c_{o}(\theta)-c_{1}(\theta)\right| .
$$

If $C_{o}, C_{1} \in B_{i}\left(S^{1}, \mathbb{R}^{2}\right)$, let

$$
d_{\infty}\left(C_{1}, C_{2}\right)=\inf _{\left\{c_{o}, c_{1} \mid \pi\left(c_{o}\right)=C_{o}, \pi\left(c_{1}\right)=C_{1}\right\}} d_{\infty}\left(c_{o}, c_{1}\right) .
$$

Lemma 3.4. For any pair $C_{1}, C_{2} \in B_{i}\left(S^{1}, \mathbb{R}^{2}\right)$,

$$
\operatorname{dist}_{G^{\rho}}\left(C_{1}, C_{2}\right) \leq d_{\infty}\left(C_{1}, C_{2}\right) \cdot \max \left\{\rho\left(\ell_{1}\right), \rho\left(\ell_{2}\right)\right\}
$$

where $\ell_{i}=\ell\left(C_{i}\right), i=1,2$.

Proof. Let $c_{1}, c_{2}$ be lifts of $C_{1}, C_{2}$ to $\operatorname{Imm}\left(S^{1}, \mathbb{R}^{2}\right)$. Let $c(t, \theta)=(1-t) c_{1}(\theta)+t c_{2}(\theta)$ be a path connecting them. Then $\left|c_{\theta}(t)\right| \leq(1-t)\left|c_{1, \theta}\right|+t\left|c_{2, \theta}\right|$ and hence, $\ell(c(t)) \leq$ $\max \left\{\ell\left(C_{1}\right), \ell\left(C_{2}\right)\right\}$. Moreover, $c_{t}=c_{2}-c_{1}$. Therefore,

$$
\begin{aligned}
\operatorname{dist}_{G^{\rho}}\left(C_{1}, C_{2}\right) & \leq \inf _{c} L_{G^{\rho}}(c) \\
& \leq \inf _{\left\{\text {pairs } c_{1}, c_{2}\right\}}\left\{\sup _{\theta}\left|c_{1}(\theta)-c_{2}(\theta)\right|\right\} \max \left\{\rho\left(\ell_{1}\right), \rho\left(\ell_{2}\right)\right\} .
\end{aligned}
$$

The polygonal approximations used in the proof of the theorem lie on the boundary of $\operatorname{Imm}\left(S^{1}, \mathbb{R}^{2}\right)$ and $B_{i}\left(S^{1}, \mathbb{R}^{2}\right)$, and Lemma 3.4 extends to them.

Proof of Theorem 3.1. Consider a path $c(t, \theta)$ connecting $C$ and $C^{\prime}$. Since the absolute curvature of the curves $c(t, \cdot)$ is uniformly bounded by a constant $K$, each curve has a tubular neighborhood of width that is bounded from below. Choose $\epsilon$ and a sequence

$$
0=t_{0}<t_{1}<\cdots<t_{N-1}<t_{N}=1
$$


such that $\epsilon K<<1$ and, for $0 \leq k<N, c\left(t_{k+1}, \cdot\right)$ is in a local chart of $c\left(t_{k}, \cdot\right)$ :

$$
c\left(t_{k+1}, \theta\right)=c\left(t_{k}, \theta\right)+f_{k}(\theta) n_{k},
$$

where $c\left(t_{k}, \cdot\right)$ is parametrized by the arclength, $n_{k}$ is the normal vector field of $c\left(t_{k}, \cdot\right)$ and $\left|f_{k}\right|<\epsilon$. Let $F=\max \left\{\left\|f_{k}^{\prime}(\theta)\right\|_{\infty} \mid 0 \leq k<N\right\}$. Let $C_{k}$ denote $\pi\left(c\left(t_{k}, \cdot\right)\right)$. Let $\ell_{k}=\ell\left(C_{k}\right)$. Let $\tilde{\ell}_{k}=(1+\epsilon K+F) \ell_{k}$.

Choose $\delta$ such that

$$
\max _{k} \sqrt{\tilde{\ell}_{k} \rho\left(\tilde{\ell}_{k}\right)} \cdot F \delta<\frac{\epsilon}{N}
$$

We now estimate the distances $\operatorname{dist}_{G \rho}\left(C_{k}, C_{k+1}\right)$. Consider the path segment $\left[c_{o}, c_{1}\right]$ from $c_{o}$ to $c_{1}$ in the local chart at $c_{o}$. Divide the range of $\theta$ into intervals of length $\delta$. Replace $f_{o}$ by a piecewise constant function $\bar{f}_{o}$ whose value in each subinterval equals the average of $f_{o}$ over that interval. Let $\bar{C}_{o}$ be the curve defined by $\bar{f}_{o}$. The Frechét distance between $C_{1}$ and $\bar{C}_{o}$ is $\leq F \delta$. The sum of the jumps in $\bar{f}_{o}$ is $\leq F \ell_{o}$. Since $\left|\bar{c}_{o}^{\prime}\right|=\left|1-\epsilon \kappa_{o}\right| \leq 1+\epsilon K, \ell\left(\bar{C}_{o}\right) \leq(1+\epsilon K+F) \ell_{o}=\tilde{\ell}_{o}$. Therefore,

$$
\operatorname{dist}_{G^{\rho}}\left(C_{1}, \bar{C}_{o}\right) \leq \max \left\{\tilde{\ell}_{o} \rho\left(\tilde{\ell}_{o}\right), \tilde{\ell}_{1} \rho\left(\tilde{\ell}_{1}\right)\right\} \cdot F \delta \leq \frac{\epsilon}{N} .
$$

Let $\alpha\left(\left[c_{o}, c_{1}\right]\right)$ denote the area swept out by the path $c$ during $\left[0, t_{1}\right]$. The area between $C_{o}$ and $\bar{C}_{o}$ equals the area between $C_{o}$ and $C_{1}$ which in turn is less than or equal to $\alpha\left(\left[c_{o}, c_{1}\right]\right)$. The curve $\bar{C}_{o}$ consists of a series of bumps over $C_{o}$. Traverse the bumps sequentially, taking care to retract the common edge of each bump with the previous bump before going to the next bump. Retracting a common edge can be done without incurring any cost.

By Lemma 3.3, if $\rho=\ell$,

$$
\operatorname{dist}_{G^{\rho}}\left(C_{o}, \bar{C}_{o}\right) \leq\left(\frac{1+\epsilon K}{1-\epsilon K}\right)^{2} \alpha\left(\left[c_{o}, c_{1}\right]\right)
$$

and if $\rho=e^{A \ell}$,

$$
\begin{gathered}
\operatorname{dist}_{G^{\rho}}\left(C_{o}, \bar{C}_{o}\right) \leq\left(\frac{1+\epsilon K}{1-\epsilon K}\right)^{3 / 2} e^{A\left(\ell_{o}+2 \epsilon\right) / 2} \sqrt{A e^{\frac{1+\epsilon K}{1-\epsilon K}}} \alpha\left(\left[c_{o}, c_{1}\right]\right) \\
\operatorname{dist}_{G^{\rho}}\left(C_{o}, C_{1}\right) \leq \operatorname{dist}_{G^{\rho}}\left(C_{o}, \bar{C}\right)+\frac{\epsilon}{N} .
\end{gathered}
$$

Similar estimates hold for $\operatorname{dist}_{G^{\rho}}\left(C_{k}, C_{k+1}\right)$ for $0<k<N$.

Therefore, if $\rho=\ell$,

$$
\operatorname{dist}_{G^{\rho}}\left(C, C^{\prime}\right) \leq\left(\frac{1+\epsilon K}{1-\epsilon K}\right)^{2} \alpha(c)+\epsilon
$$

and if $\rho=e^{A \ell}$,

$$
\operatorname{dist}_{G^{\rho}}\left(C, C^{\prime}\right) \leq\left(\frac{1+\epsilon K}{1-\epsilon K}\right)^{3 / 2} e^{A \ell_{\max }(c) / 2} \sqrt{A e^{\frac{1+\epsilon K}{1-\epsilon K}}} \alpha(c)+\epsilon .
$$

Since $\epsilon$ is arbitrary, we have

$$
\operatorname{dist}_{G^{\rho}}(C, E) \leq\left\{\begin{array}{l}
\alpha(c) \text { if } \rho(\ell)=\ell \\
e^{A \ell_{\max }(c) / 2 \sqrt{A e}} \alpha(c) \text { if } \rho(\ell)=e^{A \ell} .
\end{array}\right.
$$


For any oriented curve $C^{\text {or }}$, define the integer-valued measurable function $w_{C}$ on $\mathbb{R}^{2}$ by

$$
w_{C}(x, y)=\text { the winding number of } C \text { around }(x, y)
$$

and let

$$
d^{b}\left(C_{1}^{o r}, C_{2}^{o r}\right)=\int_{\mathbb{R}^{2}}\left|w_{C_{1}}-w_{C_{2}}\right| d x d y
$$

It is shown in [2] that for any two oriented curves $C_{1}^{o r}, C_{2}^{o r}$,

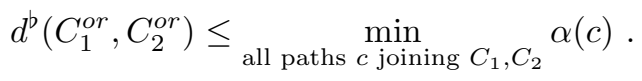

Therefore, we have

$$
\operatorname{dist}_{G^{\rho}}\left(C_{1}^{o r}, C_{2}^{o r}\right) \geq\left\{\begin{array}{l}
d^{\mathrm{b}}\left(C_{1}^{o r}, C_{2}^{o r}\right) \text { if } \rho(\ell)=\ell \\
\sqrt{A e} d^{\mathrm{b}}\left(C_{1}^{o r}, C_{2}^{o r}\right) \text { if } \rho(\ell)=e^{A \ell} .
\end{array}\right.
$$

Corollary 3.5 (Existence of minimal geodesics). If $\rho=\ell$, then the only minimal geodesics are the horizontal paths along which $\left|c_{t}^{\perp}\right| \ell$ is constant.

Proof. If $c(t)$ is a horizontal path projecting to a minimal geodesic, then $L_{G^{\rho}}(c)=$ $\alpha(c)$. Hence, since the inequality

$$
L_{G^{\rho}}(c) \geq \int_{0}^{1}\left[\int_{S^{1}}\left|c_{t}^{\perp}\right|\left|c_{\theta}\right| d \theta\right] d t=\alpha(c)
$$

is an equality if and only if $\left|c_{t}^{\perp}\right|$ does not depend on $\theta$, that is, $\frac{\partial}{\partial \theta}\left|c_{t}^{\perp}\right|=0$ (the case of "grassfire"), $\left|c_{t}^{\perp}\right|$ must be independent of $\theta$. Let $c(t, \theta)$ be a horizontal path connecting $C_{1}, C_{2}$ such that $\left|c_{t}^{\perp}\right|$ is independent of $\theta$. After reparametrization if necessary, we may assume that $c_{t} \cdot c_{\theta}=0$. Following [2], we let $w(c)$ be the 2 -current defined by the path $c(t, \theta)$. Since $c(t, \theta)$ is an immersion,

$$
d^{\mathrm{b}}\left(C_{1}^{o r}, C_{2}^{o r}\right)=\int_{\mathbb{R}^{2}}|w(c)| d x d y=\int_{S^{1} \times[0,1]}|\operatorname{det} d c(t, \theta)| d \theta d t=\alpha(c) .
$$

Therefore, $L_{G^{\rho}}(c)$ is the minimal distance between $C_{1}, C_{2}$. For $c(t, \theta)$ to be a geodesic path, reparametrize $t$ such that the infinitesimal arclength $\left|c_{t}^{\perp}\right| \ell$ is constant along the path.

Corollary 3.6. Suppose $\rho=e^{A \ell}$ and $c(t, \theta), 0 \leq t \leq 1$, is a path connecting $C_{1}, C_{2}$. Assume that $\left|c_{t}^{\perp}\right|$ does not depend on $\theta$ and $\ell(t)<1 / A$ for all $t$. Then,

$$
\operatorname{dist}_{G^{\rho}}\left(C_{1}^{o r}, C_{2}^{o r}\right)=\sqrt{A e} d^{b}\left(C_{1}^{o r}, C_{2}^{o r}\right) .
$$

It follows that the path $c(t, \theta)$ is not minimal.

Proof. Break up the interval $[0,1]$ into small segments of length $\epsilon$ and apply Lemma 3.3 with $\delta=\ell_{o}$. (The proof of the lemma extends to this case after minor modifications.) Calculate the length of the new path $\tilde{c}_{\epsilon}$ by applying the construction of Lemma 3.3 to each of the segments. We get $\lim _{\epsilon \rightarrow 0} L_{G^{\rho}}\left(\tilde{c}_{\epsilon}\right)=\sqrt{A e} \alpha(c)$ as in the proof of Theorem 3.1. 
On the other hand,

$$
\begin{aligned}
L_{G^{\rho}}(c) & =\int_{0}^{1} \sqrt{\frac{\rho(c)}{\ell(c)}}\left[\int_{S^{1}}\left|c_{t}^{\perp}\right|\left|c_{\theta}\right| d \theta\right] d t \\
& >\sqrt{\min _{\ell} \frac{\rho}{\ell}} \alpha(c)=\sqrt{A e} \alpha(c) .
\end{aligned}
$$

4. Geodesic equation. With $\psi=\frac{1}{2} \log \rho$, we have the following relation between the Levi-Civita connections [1]:

$$
\nabla_{X}^{\rho} Y=\nabla_{X}^{o} Y+\left(D_{X} \psi\right) Y+\left(D_{Y} \psi\right) X-G^{o}(X, Y) \nabla^{o} \psi
$$

Let $c(t)$ be a horizontal path in $\operatorname{Imm}\left(S^{1}, \mathbb{R}^{2}\right)$ projecting to a path in $B_{i}\left(S^{1}, \mathbb{R}^{2}\right)$. $c_{t}$ is a horizontal vector field along $c$ projecting onto $B_{i}\left(S^{1}, \mathbb{R}^{2}\right)$. Write $c_{t}=a n_{c}$. Then the geodesic curvature $\nabla_{c_{t}}^{o} c_{t}$ is horizontal and is given by the formula [2]:

$$
\nabla_{c_{t}}^{o} c_{t}=\left(a_{t}-\frac{1}{2} \kappa_{c} a^{2}\right) n_{c}
$$

Therefore,

$$
\nabla_{c_{t}}^{\rho} c_{t}=\left(a_{t}-\frac{1}{2} \kappa_{c} a^{2}+2 \psi_{t} a-\left\|a n_{c}\right\|^{2}\left(\nabla^{o} \psi \cdot n_{c}\right)\right) n_{c}
$$

The geodesic equation may be written as

$$
a_{t}=\frac{1}{2} \kappa_{c} a^{2}-2 \psi_{t} a+\left\|a n_{c}\right\|_{o}^{2}\left(\nabla^{o} \psi \cdot n_{c}\right)
$$

Suppose $\rho$ is a function of $\ell$ alone: $\rho=\rho(\ell)$. Let $\rho^{\prime}$ denote $d \rho / d \ell$. Let $\overline{f g}=\frac{1}{\ell} \int_{S^{1}} f g\left|c_{\theta}\right| d \theta$. Then

$$
\begin{aligned}
\psi_{t} & =\frac{\rho^{\prime}}{2 \rho} \ell_{t}=-\frac{\rho^{\prime} \ell}{2 \rho} \overline{a \kappa_{c}} \\
\nabla^{o} \psi \cdot n_{c} & =\frac{\rho^{\prime}}{2 \rho}\left(\nabla^{o} \ell \cdot n_{c}\right)=-\frac{\rho^{\prime}}{2 \rho} \kappa_{c} .
\end{aligned}
$$

Therefore the geodesic equation in the metric $G^{\rho(\ell)}$ is

$$
a_{t}=\frac{\kappa_{c}}{2}\left(a^{2}-\frac{\rho^{\prime} \ell}{\rho} \overline{a^{2}}\right)+a \frac{\rho^{\prime} \ell}{\rho} \overline{a \kappa_{c}} .
$$

Specifically,

$$
a_{t}=\left\{\begin{array}{ll}
\frac{\kappa_{c}}{2}\left(a^{2}-\overline{a^{2}}\right)+a \cdot \overline{a \kappa_{c}} & \text { if } \rho(\ell)=\ell, \\
\frac{\kappa_{c}}{2}\left(a^{2}-(A \ell) \overline{a^{2}}\right)+(A \ell) a \cdot \overline{a \kappa_{c}} & \text { if } \rho(\ell)=e^{A \ell} .
\end{array} .\right.
$$

As an example, consider the case of concentric circles, $c(t, \theta)=r(t) e^{i \theta}, r_{o}=r(0), r_{1}=$ $r(1)$. We have $\kappa_{c}=1 / r$ and $a=-r_{t}$. Substituting these in Eq. (1) when $\rho(\ell)=\ell$, we get $-r_{t t}=r_{t}^{2} / r$ or $\left(r^{2}\right)_{t t}=0$. Therefore, $r^{2}(t)=t r_{1}^{2}+(1-t) r_{o}^{2}$. This example is a special case of the curve evolution by "grassfire" in which $a$ is independent of $\theta$. We have $a^{2}=\overline{a^{2}}$ and the equation of the geodesic reduces to $a_{t}=a \cdot \overline{a \kappa_{c}}=-a \ell_{t} / \ell$; hence $(a \ell)_{t}=0$. Therefore, $a \ell=$ a constant. By substituting in the equation for the length of the geodesic, we find that $a \ell=$ the length of the geodesic. When $\rho(\ell)=e^{A \ell}$, the 
equation of the geodesic in the case of concentric circles is $\left(r^{2}\right)_{t t}=r_{t}^{2}(1-2 \pi r A)$, which is zero when the perimeter of the circle equals $1 / A$, marking the unique inflection point of the function $r^{2}(t)$.

5. Sectional curvature. We will use the local chart on $B_{i}\left(S^{1}, \mathbb{R}^{2}\right)$ constructed in [2]. Let $c \varepsilon \operatorname{Imm}\left(S^{1}, \mathbb{R}^{2}\right)$ be a smooth free immersion, $c: S^{1} \rightarrow \mathbb{R}^{2}$. Let $C=\pi(c) \varepsilon B_{i}\left(S^{1}, \mathbb{R}^{2}\right)$. Let $c$ be parametrized by the arclength so that $\theta$ parametrizes the scaled circle $S_{\ell}^{1}$, where $\ell$ is the length of $c$. A local chart centered at $C$ is as follows:

$$
\begin{aligned}
\Xi: C^{\infty}\left(S_{\ell}^{1},(-\epsilon, \epsilon)\right) & \rightarrow \operatorname{Imm}\left(S^{1}, \mathbb{R}^{2}\right) \\
\Xi(f)(\theta) & =c(\theta)+f(\theta) n_{c}(\theta) \\
\pi \circ \Xi: C^{\infty}\left(S_{\ell}^{1},(-\epsilon, \epsilon)\right) & \rightarrow B_{i, f}\left(S^{1}, \mathbb{R}^{2}\right) .
\end{aligned}
$$

For $h \in C^{\infty}\left(S_{\ell}^{1}, \mathbb{R}\right), h \cdot n_{c} \in T_{\Xi(f)} \operatorname{Imm}\left(S^{1}, \mathbb{R}^{2}\right)$. If $u$ is a function on $S_{\ell}^{1}$, let $u^{\prime}$ denote its derivative $d u / d \theta$. We have the following formulae from [2]:

$$
\begin{aligned}
\Xi(f)^{\prime}= & \left(1-f \kappa_{c}\right) c^{\prime}+f^{\prime} n_{c} \\
\left|\Xi(f)^{\prime}\right|= & 1-f \kappa_{c}+\frac{1}{2} f^{\prime 2}+O\left(f^{3}\right) \\
& \ell_{f}=\int_{S_{\ell}^{1}} \sqrt{\left(1-f \kappa_{c}\right)^{2}+f^{\prime 2}} d \theta=\int_{S_{\ell}^{1}}\left(1-f \kappa_{c}+\frac{1}{2} f^{\prime 2}\right) d \theta+O\left(f^{3}\right) \\
& \kappa_{f}=\kappa_{c}+\left(f^{\prime \prime}+f \kappa_{c}^{2}\right)+\left(f^{2} \kappa_{c}^{3}+\frac{1}{2} f^{\prime 2} \kappa_{c}+f f^{\prime} \kappa_{c}^{\prime}+2 f f^{\prime \prime} \kappa_{c}\right)+O\left(f^{3}\right) \\
& R_{C}^{o}(m, h, m, h)=\frac{1}{2} \|\left(m^{\prime} h-m h^{\prime} \|^{2} .\right.
\end{aligned}
$$

The conformal change in the Riemann $(4,0)$ curvature tensor is given by the formula [1]:

$$
\begin{aligned}
R^{\rho} & =\rho\left(R^{o}-G^{o} \otimes \Omega\right) \\
\Omega & =\left(\nabla^{o} d \psi-d \psi \circ d \psi+\frac{1}{2}\|d \psi\|^{2} G^{o}\right),
\end{aligned}
$$

where $₫$ denotes the Kulkarni-Nomizu product of symmetric 2-tensors:

$$
u \oplus v(x, y, z, t)=u(x, z) v(y, t)+u(y, t) v(x, z)-u(x, t) v(y, z)-u(y, z) v(x, t),
$$

$\nabla^{o} d \psi$ is the Hessian of $\psi$ with respect to $G^{o}$, and o denotes the symmetric product of symmetric tensors:

$$
d \psi \circ d \psi(x, y)=d \psi(x) d \psi(y)
$$

Let $m, h \in C^{\infty}\left(S_{\ell}^{1}, \mathbb{R}\right)$ be constant tangent vectors in the local chart that are orthonormal with respect to $G^{\rho}$. Let $\langle u, v\rangle$ denote $G^{o}(u, v)$. Note that $\|m\|^{2}=\|h\|^{2}=1 / \rho$ and $\langle m, h\rangle=0$. From (5.1), we obtain a formula for the sectional curvature:

$$
K_{B_{i}, C}^{\rho}(m, h)=\frac{\rho}{2}\left\|\left(m^{\prime} h-m h^{\prime}\right)\right\|^{2}-\Omega_{c}(m, m)-\Omega_{c}(h, h) .
$$

An explicit expression for the Hessian is

$$
\left(\nabla^{o} d \psi\right)_{c}(m, m)=D_{c, m}\left(D_{c, m} \psi\right)-D_{\Gamma^{o}(m, m)} \psi,
$$


where the Christoffel symbol $\Gamma^{o}(m, m)=-\frac{1}{2} \kappa_{c} m^{2}$ as shown in [2]. (Note that the sign convention in this paper is opposite to that used in [2].) Therefore,

$$
\Omega_{c}(m, m)=D_{c, m}\left(D_{c, m} \psi\right)+\left\langle\frac{1}{2} \kappa_{c} m^{2}, \nabla^{o} \psi\right\rangle-\left\langle m, \nabla^{o} \psi\right\rangle^{2}+\frac{1}{2 \rho}\left\|\nabla^{o} \psi\right\|^{2} .
$$

Since

$$
\nabla^{o} \psi=\frac{1}{2 \rho} \nabla^{o} \rho
$$

and

we have

$$
D_{c, m}\left(D_{c, m} \psi\right)=\frac{1}{2 \rho} D_{c, m}\left(D_{c, m} \rho\right)-\frac{1}{2 \rho^{2}}\left\langle m, \nabla^{o} \psi\right\rangle^{2}
$$

$$
\begin{aligned}
\Omega_{c}(m, m)= & \frac{1}{2 \rho} D_{c, m}\left(D_{c, m} \rho\right)+\frac{1}{4 \rho}\left\langle\kappa_{c} m^{2}, \nabla^{o} \rho\right\rangle-\frac{3}{4 \rho^{2}}\left\langle m, \nabla^{o} \rho\right\rangle^{2} \\
& +\frac{1}{8 \rho^{3}}\left\|\nabla^{o} \rho\right\|^{2} .
\end{aligned}
$$

5.1. Case when $\rho$ is a function of $\ell$ alone: $\rho=\rho(\ell)$. We compute the various quantities involved in the expression for the sectional curvature using the local chart.

$$
\begin{aligned}
D_{c, m} \ell & =-\left\langle m, \kappa_{c}\right\rangle \\
\nabla^{o} \rho & =-\rho^{\prime} \kappa_{c} \\
D_{c, m}\left(D_{c, m} \rho\right) & =\rho^{\prime} D_{c, m}\left(D_{c, m} \ell\right)+\rho^{\prime \prime}\left(D_{c, m} \ell\right)^{2} \\
& =\rho^{\prime}\left\|m^{\prime}\right\|^{2}+\rho^{\prime \prime}\left\langle m, \kappa_{c}\right\rangle^{2} \\
\left\langle\kappa_{c} m^{2}, \nabla^{o} \rho\right\rangle & =-\rho^{\prime}\left\|m \kappa_{c}\right\|^{2} \\
\left\langle m, \nabla^{o} \rho\right\rangle^{2} & =\rho^{\prime 2}\left\langle m, \kappa_{c}\right\rangle^{2} \\
\left\|\nabla^{o} \rho\right\|^{2} & =\rho^{\prime 2}\left\|\kappa_{c}\right\|^{2} \\
\Omega_{c}(m, m) & =\frac{\rho^{\prime}}{2 \rho}\left\|m^{\prime}\right\|^{2}-\frac{\rho^{\prime}}{4 \rho}\left\|m \kappa_{c}\right\|^{2}-\frac{3 \rho^{\prime 2}-2 \rho \rho^{\prime \prime}}{4 \rho^{2}}\left\langle m, \kappa_{c}\right\rangle^{2}+\frac{\rho^{\prime 2}}{8 \rho^{3}}\left\|\kappa_{c}\right\|^{2} .
\end{aligned}
$$

Substituting the explicit expressions in the formula for the sectional curvature, we get

$$
\begin{aligned}
& K_{B_{i}, C}^{\rho(l)}(m, h)=\frac{\rho}{2}\left\|m^{\prime} h-m h^{\prime}\right\|^{2}-\frac{\rho^{\prime}}{2 \rho}\left(\left\|m^{\prime}\right\|^{2}+\left\|h^{\prime}\right\|^{2}\right) \\
& +\frac{\rho^{\prime}}{4 \rho}\left(\left\|m \kappa_{c}\right\|^{2}+\left\|h \kappa_{c}\right\|^{2}\right) \\
& +\frac{3 \rho^{\prime 2}-2 \rho \rho^{\prime \prime}}{4 \rho^{2}}\left(\left\langle m, \kappa_{c}\right\rangle^{2}+\left\langle h, \kappa_{c}\right\rangle^{2}\right)-\frac{\rho^{\prime 2}}{4 \rho^{3}}\left\|\kappa_{c}\right\|^{2} .
\end{aligned}
$$

Each of the last three terms on the right-hand side is absolutely bounded with respect to $m$ and $h$ since:

$$
\left\|m \kappa_{c}\right\|^{2}+\left\|h \kappa_{c}\right\|^{2} \leq \frac{2\left\|\kappa_{c}\right\|_{\infty}^{2}}{\rho}, \quad\left\langle m, \kappa_{c}\right\rangle^{2}+\left\langle h, \kappa_{c}\right\rangle^{2} \leq \frac{2\left\|\kappa_{c}\right\|^{2}}{\rho}
$$

where $\left\|\kappa_{c}\right\|_{\infty}=\max _{\theta}\left|\kappa_{c}(\theta)\right|$. Therefore, the boundedness of the sectional curvature from above depends on the first two terms. For a fixed $m$, the magnitude of each of the two 
terms depends on $\left\|h^{\prime}\right\|$, which can be made arbitrarily large while keeping $\|h\|$ fixed by making $h$ highly oscillatory.

Proposition 5.1. Let $m$ be a tangent vector at $C \epsilon B_{i}$. Then the sectional curvature $K_{B_{i}, C}^{\rho(l)}(m, h)$ is uniformly bounded from above with respect to $h$ if and only if $m^{2}(\theta) \leq$ $\frac{\rho^{\prime} \ell}{\rho} \overline{m^{2}}$.

Proof. We may assume that $m, h$ are orthonormal with respect to the metric $G^{\rho}$ so that $\frac{\rho^{\prime} \ell}{\rho} \overline{m^{2}}=\frac{\rho^{\prime}}{\rho^{2}}$. We need to estimate only the first two terms on the right-hand side of Eq. (5.3).

Suppose $\|m\|_{\infty}^{2} \leq \frac{\rho^{\prime}}{\rho^{2}}$. Then

$$
\begin{aligned}
& \frac{\rho}{2}\left\|m^{\prime} h-m h^{\prime}\right\|^{2}-\frac{\rho^{\prime}}{2 \rho}\left(\left\|m^{\prime}\right\|^{2}+\left\|h^{\prime}\right\|^{2}\right) \\
& =\rho \int_{S_{\ell}^{1}}\left[\frac{\rho}{2}\left(m^{\prime} h\right)^{2}-\frac{\rho^{\prime}}{2 \rho} m^{\prime 2}-\rho m m^{\prime} h h^{\prime}+\frac{\rho}{2}\left(m^{2}-\frac{\rho^{\prime}}{\rho^{2}}\right) h^{\prime 2}\right] d \theta \\
& \leq \frac{\rho}{2}\left\|m^{\prime}\right\|_{\infty}^{2}-\frac{\rho^{2}}{4} \int_{S_{\ell}^{1}}\left(m^{2}\right)^{\prime}\left(h^{2}\right)^{\prime} d \theta \\
& \leq \frac{\rho}{2}\left\|m^{\prime}\right\|_{\infty}^{2}+\frac{\rho^{2}}{4} \int_{S_{\ell}^{1}}\left(m^{2}\right)^{\prime \prime}\left(h^{2}\right) d \theta \\
& \leq \frac{\rho}{2}\left\|m^{\prime}\right\|_{\infty}^{2}+\frac{\rho}{4}\left\|\left(m^{2}\right)^{\prime \prime}\right\|_{\infty}<\infty .
\end{aligned}
$$

Conversely, suppose $\|m\|_{\infty}^{2}>\frac{\rho^{\prime}}{\rho^{2}}$. Choose $\epsilon$ such that $U=\left\{\theta: m^{2}(\theta)>\rho^{\prime} / \rho^{2}+\epsilon\right\}$ is not empty. Let $h$ be a high-frequency wave function with $\sup (h) \subset U$. Then

$$
\begin{aligned}
& \frac{\rho}{2}\left\|m^{\prime} h-m h^{\prime}\right\|^{2}-\frac{\rho^{\prime}}{2 \rho}\left(\left\|m^{\prime}\right\|^{2}+\left\|h^{\prime}\right\|^{2}\right) \\
& =\rho \int_{S_{\ell}^{1}}\left[\frac{\rho}{2}\left(m^{\prime} h\right)^{2}-\frac{\rho^{\prime}}{2 \rho} m^{\prime 2}-\rho m m^{\prime} h h^{\prime}+\frac{\rho}{2}\left(m^{2}-\frac{\rho^{\prime}}{\rho^{2}}\right) h^{\prime 2}\right] d \theta \\
& \geq-\frac{\rho^{\prime}}{2 \rho}\left\|m^{\prime}\right\|^{2}+\frac{\rho^{2}}{4} \int_{S_{\ell}^{1}}\left(m^{2}\right)^{\prime \prime}\left(h^{2}\right) d \theta+\frac{\rho \epsilon}{2}\left\|h^{\prime}\right\|^{2} \\
& \geq-\frac{\rho^{\prime}}{2 \rho}\left\|m^{\prime}\right\|^{2}-\frac{\rho^{2}}{4}\left\|\left(m^{2}\right)^{\prime \prime}\right\|_{\infty}+\frac{\rho \epsilon}{2}\left\|h^{\prime}\right\|^{2}
\end{aligned}
$$

which tends to $\infty$ as the frequency of the wave function $h$ tends to $\infty$.

If $U=\left\{\theta: m^{2}(\theta)>\rho^{\prime} / \rho^{2}+\epsilon\right\}$ is not empty, then

$$
\begin{aligned}
1 & =\rho\left[\int_{U} m^{2} d \theta+\int_{[0, \ell] / U} m^{2} d \theta\right] \\
& \geq \rho \int_{U} m^{2} d \theta \geq\left(\frac{\rho^{\prime}}{\rho}+\epsilon \rho\right)|U|
\end{aligned}
$$


and hence $|U|<\rho / \rho^{\prime}$. If $\rho=\ell,|U|<\ell$ and if $\rho=e^{A \ell},|U|<1 / A$. Thus, the case when $\|m\|_{\infty}^{2}>\rho^{\prime} / \rho^{2}$ may be seen as a generalization of the rectangular bump considered in $\S 3$.

If $\rho=\ell$, then the sectional curvature is bounded if and only if $m^{2}(\theta) \leq \overline{m^{2}}$, which is true if and only if $m=1 / \ell$. Setting $m=1 / \ell$, we get

$$
K_{B_{i}, C}^{\rho(l)}(m, h)=\frac{\left\|h \kappa_{c}\right\|^{2}}{4 \ell}+\frac{3}{4 \ell^{2}}\left(\left\langle\frac{1}{\ell}, \kappa_{c}\right\rangle^{2}+\left\langle h, \kappa_{c}\right\rangle^{2}\right)
$$

which is always positive. If $h$ and $\kappa_{c}$ additionally have disjoint supports, then the sectional curvature equals $3 \pi^{2} N^{2} / \ell^{4}$, where $N$ is the rotation index of $C$.

If $\rho=e^{A \ell}$, then the sectional curvature is bounded if and only if $m^{2}(\theta) \leq(A \ell) \overline{m^{2}}$. In particular, the sectional curvature is unbounded for every $m$ if $\ell<1 / A$.

For an example of a negative sectional curvature, consider the unit square with slightly rounded corners. Choose $m, h$ such that $\sup (m)$ and $\sup (h)$ are disjoint and concentrated along the straight portions of the square. Then

$$
K_{B_{i}, C}^{\rho(l)}(m, h)=-\frac{\rho^{\prime}}{2 \rho}\left(\left\|m^{\prime}\right\|^{2}+\left\|h^{\prime}\right\|^{2}\right)-\frac{\rho^{2}}{4 \rho^{3}}\left\|\kappa_{c}\right\|^{2}
$$

5.2. Case when $\rho=\int_{S^{1}} \varphi\left(\kappa^{2}\right)\left|c_{\theta}\right| d \theta$. We again assume that the tangent vectors $m, h \in$ $C^{\infty}\left(S_{\ell}^{1}, \mathbb{R}\right)$ are constant in the local chart and are orthonormal with respect to $G^{\rho}$.

$$
\begin{aligned}
D_{c, m}\left|c_{\theta}\right| & =-\kappa_{c} m \\
D_{c, m}\left(D_{c, m}\left|c_{\theta}\right|\right) & =m^{\prime 2} \\
D_{c, m} \kappa & =m^{\prime \prime}+\kappa_{c}^{2} m \\
D_{c, m}\left(D_{c, m} \kappa\right) & =2 \kappa_{c}^{3} m^{2}+\kappa_{c} m^{\prime 2}+2 \kappa_{c}^{\prime} m m^{\prime}+4 \kappa_{c} m m^{\prime \prime} \\
D_{c, m} \rho & =\int_{S^{1}}\left[2 \kappa_{c} \varphi^{\prime}\left(m^{\prime \prime}+\kappa_{c}^{2} m\right)-\varphi \kappa_{c} m\right]\left|c_{\theta}\right| d \theta \\
& =\int_{S^{1}}\left[\left(2 \kappa_{c} \varphi^{\prime}\right)^{\prime \prime}+2 \kappa_{c}^{3} \varphi^{\prime}-\kappa_{c} \varphi\right] m\left|c_{\theta}\right| d \theta
\end{aligned}
$$

Therefore,

$$
\begin{aligned}
\nabla^{o} \rho= & \left(2 \kappa_{c} \varphi^{\prime}\right)^{\prime \prime}+2 \kappa_{c}^{3} \varphi^{\prime}-\kappa_{c} \varphi \\
D_{c, m}\left(D_{c, m} \rho\right)= & \int_{S^{1}}\left[2 \kappa_{c} \varphi^{\prime}\left(4 \kappa_{c} m m^{\prime \prime}+2 \kappa_{c}^{\prime} m m^{\prime}+\kappa_{c} m^{\prime 2}+2 \kappa_{c}^{3} m^{2}\right)\right. \\
& +2\left(2 \kappa_{c}^{2} \varphi^{\prime \prime}+\varphi^{\prime}\right)\left(m^{\prime \prime}+\kappa_{c}^{2} m\right)^{2} \\
& \left.-4 \kappa_{c}^{2} \varphi^{\prime} m\left(m^{\prime \prime}+\kappa_{c}^{2} m\right)+\varphi m^{\prime 2}\right]\left|c_{\theta}\right| d \theta \\
= & \int_{S^{1}}\left[2\left(2 \kappa_{c}^{2} \varphi^{\prime \prime}+\varphi^{\prime}\right) m^{\prime \prime 2}+8 \kappa_{c}^{2}\left(\kappa_{c}^{2} \varphi^{\prime \prime}+\varphi^{\prime}\right) m m^{\prime \prime}\right. \\
& +\left(2 \kappa_{c}^{2} \varphi^{\prime}+\varphi\right) m^{\prime 2}+4 \kappa_{c} \kappa_{c}^{\prime} \varphi^{\prime} m m^{\prime} \\
& \left.+2 \kappa_{c}^{4}\left(2 \kappa_{c}^{2} \varphi^{\prime \prime}+\varphi^{\prime}\right) m^{2}\right]\left|c_{\theta}\right| d \theta .
\end{aligned}
$$


Applying integration by parts to the second term, we get

$$
\begin{aligned}
D_{c, m}\left(D_{c, m} \rho\right)= & \int_{S^{1}}\left[2\left(2 \kappa_{c}^{2} \varphi^{\prime \prime}+\varphi^{\prime}\right) m^{\prime \prime 2}-\left(8 \kappa_{c}^{4} \varphi^{\prime \prime}+6 \kappa_{c}^{2} \varphi^{\prime}-\varphi\right) m^{\prime 2}\right. \\
& -4 \kappa_{c}\left(2 \kappa_{c}^{3} \varphi^{\prime \prime \prime}+2 \kappa_{c}\left(4 \kappa_{c} \kappa_{c}^{\prime}+1\right) \varphi^{\prime \prime}+3 \kappa_{c}^{\prime} \varphi^{\prime}\right) m m^{\prime} \\
& \left.+2 \kappa_{c}^{4}\left(2 \kappa_{c}^{2} \varphi^{\prime \prime}+\varphi^{\prime}\right) m^{2}\right]\left|c_{\theta}\right| d \theta .
\end{aligned}
$$

Since there exists a constant $\gamma$ such that for all $f \varepsilon C^{2}\left(S^{1}\right)$, if $0<\epsilon \leq 1$,

$$
\left\|u^{\prime}\right\|^{2} \leq \gamma\left(\epsilon\left\|u^{\prime \prime}\right\|^{2}+\frac{1}{\epsilon}\|u\|\right)^{2}
$$

boundedness of $K_{B_{i}, C}^{\rho}(m, h)$ from above at $C$ for a given $m$ is clearly controlled by the term

$$
-\frac{1}{\rho} \int_{S^{1}}\left(2 \kappa_{c}^{2} \varphi^{\prime \prime}+\varphi^{\prime}\right) h^{\prime \prime 2}\left|c_{\theta}\right| d \theta
$$

which is negative if $2 x \varphi^{\prime \prime}(x)+\varphi^{\prime}(x)>0$. If $x>0$, then

$$
\frac{1}{2 \sqrt{x}}\left(2 x \varphi^{\prime \prime}(x)+\varphi^{\prime}(x)\right)=\sqrt{x} \varphi^{\prime \prime}(x)+\frac{\varphi^{\prime}(x)}{2 \sqrt{x}}=\left(\sqrt{x} \varphi^{\prime}(x)\right)^{\prime} .
$$

Therefore, for a fixed $m, K_{B_{i}, C}^{\rho}(m, h)$ is bounded from above at $C$ if $\varphi^{\prime}(0)>0$ and $\sqrt{x} \varphi^{\prime}(x)$ is a strictly increasing function. A case of particular interest in computer vision is $\varphi(x)=(1+A x)^{\alpha}$ with $\alpha, A>0$. We then have

$$
2 x \varphi^{\prime \prime}(x)+\varphi^{\prime}(x)=\left[A \alpha(1+A x)^{\alpha-2}\right][A x(2 \alpha-1)+1]
$$

which is positive if $\alpha \geq 1 / 2$.

Acknowledgment. Suggestions by David Mumford contributed greatly to this paper.

\section{References}

[1] A. L. Besse, "Einstein manifolds", Springer-Verlag, 1987. MR867684 (88f:53087)

[2] P. Michor and D. Mumford, "Riemannian geometries on spaces of plane curves", arXiv:math.DG/0312384, v2, Sep. 22, 2004. MR2201275 (2007a:58007)

[3] P. Michor and D. Mumford, "An overview of the Riemannian metrics on spaces of curves using the Hamiltonian approach", Tech. Report, ESI Preprint \#1798, 2005.

[4] E. Klassen, A. Srivastava, W. Mio, and S. H. Joshi, "Analysis of planar shapes using geodesic paths on shape spaces", IEEE Trans. PAMI, 26(3), pp. 372-383, 2003.

[5] W. Mio and A. Srivastava, "Elastic-string models for representation and analysis of planar shapes", CVPR(2), 2004, pp. 10-15.

[6] W. Mio, A. Srivastava, and S. H. Joshi, "On shape of plane elastic curves", International Journal of Computer Vision, 73(3), pp. 307-324.

[7] A. Yezzi and A. Mennucci, "Conformal Riemannian metrics in space of curves", EUSIPCO04, MIA, 2004.

[8] A. Yezzi and A. Mennucci, "Metrics in the space of curves", arXiv:math.DG/0412454, v2, May 25, 2005.

[9] L. Younes, "Computable elastic distances between shapes," SIAM J. Appl. Math., 58 (1998), pp. 565-586. MR1617630 (99c:68221) 